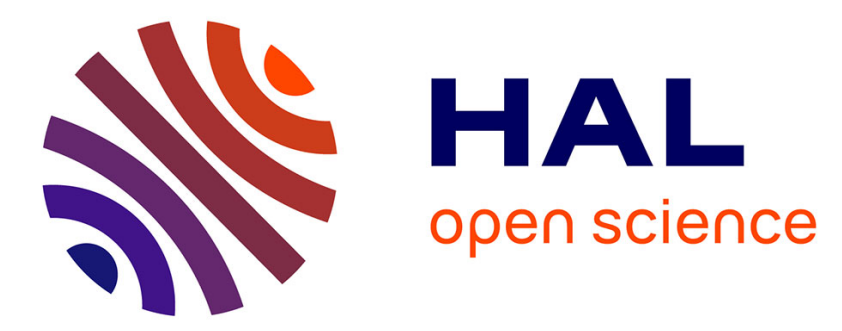

\title{
On a non-local equation arising in population dynamics
} Jérôme Coville, Louis Dupaigne

\section{To cite this version:}

Jérôme Coville, Louis Dupaigne. On a non-local equation arising in population dynamics. Proceedings of the Royal Society of Edinburgh: Section A, Mathematics, 2007, 137 (4), pp.727-755. 10.1017/S0308210504000721 . hal-02659746

\section{HAL Id: hal-02659746 \\ https: / hal.inrae.fr/hal-02659746}

Submitted on 30 May 2020

HAL is a multi-disciplinary open access archive for the deposit and dissemination of scientific research documents, whether they are published or not. The documents may come from teaching and research institutions in France or abroad, or from public or private research centers.
L'archive ouverte pluridisciplinaire HAL, est destinée au dépôt et à la diffusion de documents scientifiques de niveau recherche, publiés ou non, émanant des établissements d'enseignement et de recherche français ou étrangers, des laboratoires publics ou privés. 


\title{
On a nonlocal equation arising in population dynamics
}

\author{
Jerome Coville ${ }^{1}$ and Louis Dupaigne ${ }^{2}$ \\ E-mails: coville@ann.jussieu.fr, dupaigne@math.cnrs.fr \\ ${ }^{1}$ Laboratoire Jacques-Louis Lions \\ Université Pierre et Marie Curie \\ boîte courrier 187 \\ 75252 Paris Cedex 05 \\ France \\ ${ }^{2}$ Laboratoire Amiénois de Mathématique Fondamentale et Appliquée \\ Université Picardie Jules Verne \\ Faculté de Mathématiques et d'Informatique \\ 33, rue Saint-Leu 80039 Amiens Cedex 1 \\ France
}

June 26, 2006

\begin{abstract}
We study a one-dimensional nonlocal variant of Fisher's equation describing the spatial spread of a mutant in a given population, and its generalization to the so-called monostable nonlinearity. The dispersion of the genetic characters is assumed to follow a nonlocal diffusion law modelled by a convolution operator. We prove that as in the classical (local) problem, there exist travelling-wave solutions of arbitrary speed beyond a critical value and also characterize the asymptotic behaviour of such solutions at infinity. Our proofs rely on an appropriate version of the maximum principle, qualitative properties of solutions and approximation schemes leading to singular limits.
\end{abstract}




\section{Introduction}

In 1930, Fisher [9] suggested to model the spatial spread of a mutant in a given population by the following reaction-diffusion equation :

$$
u_{t}-\Delta u=u(1-u),
$$

where $u$ represents the gene fraction of the mutant. Dispersion of the genetic characters is assumed to follow a diffusion law while the logistic term $u(1-u)$ takes into account the saturation of this dispersion process.

Since then, much attention has been drawn to reaction-diffusion equations, as they have proved to give a robust and accurate description of a wide variety of phenomena, ranging from combustion to bacterial growth, nerve propagation or epidemiology. We point the interested reader to $[8,13,11]$ and their many references.

In this work, we consider a variant of (1.1) where diffusion is modeled by a convolution operator. Going back to the early work of Kolmogorov - PetrovskiiPiskounov (see [12]), dispersion of the gene fraction at point $y \in \mathbb{R}^{n}$ should affect the gene fraction at $x \in \mathbb{R}^{n}$ by a factor $J(x, y) u(y) d y$ where $J(x, \cdot)$ is a probability density. Restricting to a one-dimensional setting and assuming that such a diffusion process depends only on the distance between two niches of the population, we end up with the equation

$$
u_{t}-(J \star u-u)=f(u),
$$

where $J: \mathbb{R} \rightarrow \mathbb{R}$ is a nonnegative even function of mass one and for $x \in \mathbb{R}$, $J \star u(x)=\int_{\mathbb{R}} J(x-y) u(y) d y$. More precisely, we assume in what follows that

$$
J \in C^{1}(\mathbb{R}), \quad J \geq 0, \quad J(x)=J(-x) \quad \text { and } \quad \int_{\mathbb{R}} J=1,
$$

We make the additional technical assumption

$$
\exists \lambda>0, \quad \int_{\mathbb{R}} J(x) e^{\lambda x} d x<+\infty .
$$

For example, (H2) is satisfied if $J$ has compact support or if $J(x)=\frac{1}{2 \lambda} e^{-\lambda|x|}$ for some $\lambda>0$.

The nonlinearity $f$ in (1.2) can be chosen more generally than in equation (1.1). In the literature, three types of nonlinearities appear, according to the underlined application: we always assume that $f \in C^{1}(\mathbb{R}), f(0)=f(1)=0, f^{\prime}(1)<0$ and

- we say that $f$ is of bistable type if there exists $\theta \in(0,1)$ such that

$$
f<0 \text { in }(0, \theta), \quad f(\theta)=0 \text { and } f>0 \text { in }(\theta, 1)
$$

- $f$ is of ignition type if there exists $\theta \in(0,1)$ such that

$$
\left.f\right|_{[0, \theta]} \equiv 0,\left.\quad f\right|_{(\theta, 1)}>0 \quad \text { and } f(1)=0 .
$$

- $f$ is of monostable type if

$$
f>0 \text { in }(0,1)
$$


In the present article, we will focus on the monostable nonlinearity. Observe that equation (1.1) falls in this case.

(1.1) can also be seen as a first order approximation of (1.2). Indeed if any given niche of the species is assumed to interact mostly with close-by neighbours, the diffusion term is of the form $J_{\epsilon}(x):=\frac{1}{\epsilon} J\left(\frac{1}{\epsilon} x\right)$, where $J$ is compactly supported and $\epsilon>0$ is small. We then have

$$
\begin{aligned}
J_{\epsilon} \star u-u & =\frac{1}{\epsilon} \int J\left(\frac{1}{\epsilon} y\right)(u(x-y)-u(x)) d y=\int J(z)(u(x-\epsilon z)-u(x)) d z \\
& =-\epsilon \int J(z) u^{\prime}(x) z d z+\frac{1}{2} \epsilon^{2} \int z^{2} J(z) u^{\prime \prime}(x) d z+o\left(\epsilon^{2}\right)=c \epsilon^{2} u^{\prime \prime}(x)+o\left(\epsilon^{2}\right),
\end{aligned}
$$

where we used the fact that $J$ is even in the last equality.

We observe that equation (1.2) can be related to a class of problems studied in $[15,16]$. However, our approach differs in at least two ways : firstly, from the technical point of view, inverting the operator $u \rightarrow u_{t}-(J \star u-u)$ in any reasonable space yields no $a$ priori regularity property on the solution $u$ and the compactness assumptions made in [16] no longer hold in our case.

Secondly, whereas the author favored discrete models over continuous ones to describe the dynamics of certain populations, we remain interested in the latter. In particular, we have in mind the following application to adaptative dynamics : in [10], the authors study a probabilistic model describing the microscopic behavior of the evolution of genetic traits in a population subject to mutation and selection. Averaging over a large number of individuals in the initial state, they derive in the limit a deterministic equation, a special case of which can be written as

$$
\partial_{t} u=[J \star u-u]+(1-K \star u) u,
$$

where $J(x)$ is a kernel taking into account mutation about trait $x$ and $K(x)$ is a competition kernel, measuring the "intensity" of the interaction between $x$ and $y$. Taking $K(x)=\delta$, we recover equation (1.2) as a special case of (1.3).

The aim of this article is the study of so-called travelling-wave solutions of equation (1.2) i.e. solutions of the form

$$
u(x, t)=U(x+c t)
$$

where $c \in \mathbb{R}$ is called the wave speed and $U$ the wave profile, which is required to solve the equation

$$
\left\{\begin{array}{l}
{[J \star U-U]-c U^{\prime}+f(U)=0 \text { in } \mathbb{R}} \\
U(-\infty)=0 \\
U(+\infty)=1,
\end{array}\right.
$$

where $U( \pm \infty)$ denotes the limit of $U(x)$ as $x \rightarrow \pm \infty$.

Such solutions are expected to give the asymptotic behavior in large time for solutions of (1.2) with say compactly supported initial data : in the Fisher equation, 
this is equivalent to saying that the mutant propagates (after some time) at constant speed and along the profile $U$. It is therefore of interest to prove existence of such solutions.

The first results in this direction are due to Schumacher [14], who considered the monostable nonlinearity, under the extra assumption that $f(r) \geq h_{0} r-K r^{1+\alpha}$, for some $h_{0}, K, \alpha>0$ and all $r \in[0,1]$. In this case, his results imply existence of travelling waves with arbitrary speed $c \geq c^{*}$, where $c^{*}$ is the smallest $c \in \mathbb{R}$ such that $\rho_{c}: \mathbb{R} \rightarrow \mathbb{R}$ defined by

$$
\rho_{c}(\lambda)=-\lambda c+\int J(z) e^{\lambda z} d z-1+f^{\prime}(0)
$$

vanishes for some $\lambda>0$. Observe from assumption (H1) that $\int J(z) e^{\lambda z}=\int J(z) e^{-\lambda z}$. So finding $\lambda>0$ such that $\rho_{c}(\lambda)=0$ amounts to looking for an explicit solution of the form $v(x)=e^{\lambda x}$ of the equation

$$
J \star v-v-c v^{\prime}+f^{\prime}(0) v=0,
$$

obtained by linearizing (1.4) near $x=-\infty$. $v$ then yields the expected asymptotic decay near $x=-\infty$ of solutions of (1.4).

Finally, if $c>c^{*}$ and under some extra assumptions on $f$, Schumacher shows that the profile $U$ of the associated travelling wave is unique up to translation.

Recently, Carr and Chmaj [3] completed the work of Schumacher. For the "KPP" nonlinearity (i.e. if $f$ is monostable and $f(r) \leq f^{\prime}(0) r$ for all $\left.r \in[0,1]\right)$ and if $J$ has compact support, they show that the above uniqueness result can be extended to $c=c^{*}$.

Concerning the bistable nonlinearity, Bates-Fife-Ren-Wang [1] and Chen [4] showed that there exists an increasing travelling wave $U$ with speed $c$ solving (1.4). Furthermore if $V$ is another nondecreasing travelling wave with speed $c^{\prime}$ then $c=c^{\prime}$ and $V(x)=U(x+\tau)$ for some $\tau \in \mathbb{R}$.

Coville [6] then looked at the case of ignition nonlinearities and proved again the existence and uniqueness (up to translation) of an increasing travelling wave $(U, c)$. Coville also obtained the existence of at least one travelling-wave solution in the monostable case.

Our first theorem extends some of the afore-mentioned results of Schumacher to the general monostable case:

\section{Theorem 1.1.}

Assume (H1) and (H2) hold and assume that $f$ is of monostable type. Then there exists a constant $c^{*}>0$ (called the minimal speed of the travelling wave) such that for all $c \geq c^{*}$, there exists an increasing solution $U \in C^{1}(\mathbb{R})$ of (1.4) while no nondecreasing travelling wave of speed $c<c^{*}$ exists.

Our second result extends previous work of Coville [6] regarding the behavior of the travelling front $U$ near $\pm \infty$.

\section{Proposition 1.1.}

Assume (H1) and (H2) hold. Then given any travelling-wave solution (U,c) of (1.4) with $f$ monostable, the following assertions hold: 
1. There exist positive constants $A, B, M, \lambda_{0}$ and $\delta_{0}$ such that

$$
B e^{-\delta_{0} y} \leq 1-U(y) \leq A e^{-\lambda_{0} y} \text { for } y \geq M .
$$

2. If $f^{\prime}(0)>0$ then there exists positive constants $K, N$ and $\lambda_{1}$ such that

$$
U(y) \leq K e^{\lambda_{1} y} \text { for } y \leq-N .
$$

The first point is an easy consequence of a similar result when $f$ is of bistable or ignition type, proved in [6].

Regarding Theorem 1.1, our proof is based on the study of two auxiliary problems and the construction of adequate super and subsolutions. We work in three steps.

We start by showing existence and uniqueness of a solution for

$$
\left\{\begin{aligned}
\mathcal{L} u+f(u) & =-h_{r}(x) \quad \text { in } \Omega, \\
u(-r) & =\theta, \\
u(+\infty) & =1,
\end{aligned}\right.
$$

where given $\epsilon>0, r \in \mathbb{R}, c \in \mathbb{R}$ and $\theta \in(0,1)$,

$$
\begin{aligned}
& \Omega=(-r,+\infty), \\
& \mathcal{L} u=\mathcal{L}(\epsilon, r, c) u=\epsilon u^{\prime \prime}+\left[\int_{-r}^{+\infty} J(x-y) u(y) d y-u\right]-c u^{\prime}, \\
& h_{r}(x)=\theta \int_{-\infty}^{-r} J(x-y) d y .
\end{aligned}
$$

The existence is obtained via an iterative scheme using a comparison principle and appropriate sub and supersolutions.

In the second step, with a standard limiting procedure (as $r \rightarrow+\infty$ ), we prove Theorem 1.1 for the problem

$$
\left\{\begin{aligned}
\mathcal{M} u+f(u) & =0 \\
u(-\infty) & =0 \\
u(+\infty) & =1
\end{aligned}\right.
$$

where given $\epsilon>0, c \in \mathbb{R}$,

$$
\mathcal{M} u=\mathcal{M}(\epsilon, c) u=\epsilon u^{\prime \prime}+[J \star u-u]-c u^{\prime} .
$$

We stress the fact that unlike (1.5), (1.9) does not have an (increasing and smooth) solution $u$ for arbitrary values of $c \in \mathbb{R}$.

Finally, in the last step we send $\epsilon \rightarrow 0$ and extract converging subsequences.

Though elementary in nature, the proofs require a number of lemmas which we list and prove in the Appendix. We construct sub and supersolutions for (1.5) and (1.9) in Section 2. After obtaining some useful a priori estimates in Section 3, we prove existence and uniqueness of solutions of (1.5) in Section 4. In Section 5, we show the existence of a speed $c^{*}(\epsilon)>0$ such that (1.9) admits a solution for every $c \geq c^{*}(\epsilon)$. We complete the proof of Theorem 1.1 in Section 6. Section 7 is devoted to the proof of Proposition 1.1. 


\section{Existence of sub and supersolutions}

We start with the construction of a supersolution of (1.9) for speeds $c \geq \bar{\kappa}(\epsilon)$ for some $\bar{\kappa}(\epsilon)>0$.

\section{Lemma 2.1.}

Let $\epsilon>0$. There exists a real number $\bar{\kappa}(\epsilon)>0$ and an increasing function $\bar{w} \in C^{2}(\mathbb{R})$ such that, given any $c \geq \bar{\kappa}(\epsilon)$

$$
\left\{\begin{array}{l}
\mathcal{M} \bar{w}+f(\bar{w}) \leq 0 \text { in } \mathbb{R} \\
\bar{w}(-\infty)=0 \\
\bar{w}(+\infty)=1
\end{array}\right.
$$

where $\mathcal{M}=\mathcal{M}(\epsilon, c)$ is defined by (1.10). Furthermore, $\bar{w}(0)=\frac{1}{2}$.

\section{Proof:}

Fix positive constants $N, \lambda, \delta$ such that $\lambda>\delta$ and (H2) holds.

Let $\bar{w} \in C^{2}(\mathbb{R})$ be a positive increasing function satisfying

- $\bar{w}(x)=e^{\lambda x}$ for $x \in(-\infty,-N]$,

- $\bar{w}(x) \leq e^{\lambda x}$ on $\mathbb{R}$,

- $\bar{w}(x)=1-e^{-\delta x}$ for $x \in[N,+\infty)$,

- $\bar{w}(0)=\frac{1}{2}$.

Let $x_{0}=e^{-\lambda N}$ and $x_{1}=1-e^{-\delta N}$. We have $0<x_{0}<x_{1}<1$.

We now construct a positive function $g$ defined on $(0,1)$ which satisfies $g(\bar{w}) \geq f(\bar{w})$.

Since $f$ is smooth near 0 and 1 , we have for $c$ large enough, say $c \geq \kappa_{0}$,

$$
\lambda(c-\lambda) s \geq f(s) \text { for } s \in\left[0, x_{0}\right]
$$

and

$$
\delta(c-\delta)(1-s) \geq f(s) \text { for } s \in\left[x_{1}, 1\right] .
$$

Therefore we can achieve $g(s) \geq f(s)$ for $s$ in [0,1], with $g$ defined by:

$$
g(s)= \begin{cases}\lambda\left(\kappa_{0}-\lambda\right) s & \text { for } 0 \leq s \leq x_{0} \\ l(s) & \text { for } x_{0}<s<x_{1} \\ \delta\left(\kappa_{0}-\delta\right)(1-s) & \text { for } x_{1} \leq s \leq 1\end{cases}
$$

where $l$ is any smooth positive function greater than $f$ on $\left[x_{0}, x_{1}\right]$ such that $g$ is of class $C^{1}$.

According to (2.3), for $x \leq-N$ i.e. for $w \leq e^{-\lambda N}$, we have

$$
\begin{aligned}
\mathcal{M} \bar{w}+g(\bar{w}) & =\epsilon \bar{w}^{\prime \prime}+J \star \bar{w}-\bar{w}-c \bar{w}^{\prime}+g(\bar{w}) \\
& =\epsilon \lambda^{2} e^{\lambda x}+J \star \bar{w}-e^{\lambda x}-\lambda c e^{\lambda x}+\lambda\left(\kappa_{0}-\lambda\right) e^{\lambda x} \\
& \leq \epsilon \lambda^{2} e^{\lambda x}+J \star e^{\lambda x}-e^{\lambda x}-\lambda c e^{\lambda x}+\lambda\left(\kappa_{0}-\lambda\right) e^{\lambda x} \\
& \leq e^{\lambda x}\left[\int_{\mathbb{R}} J(z) e^{\lambda z} d z-1-\lambda\left(c-\kappa_{0}\right)-\lambda^{2}(1-\epsilon)\right] \\
& \leq 0,
\end{aligned}
$$


for $c$ large enough, say

$$
c \geq \kappa_{1}=\frac{\int_{\mathbb{R}} J(z) e^{\lambda z} d z-1+\lambda \kappa_{0}-\lambda^{2}(1-\epsilon)}{\lambda} .
$$

Furthermore for $\bar{w} \geq 1-e^{-\delta N}$ we have,

$$
\begin{aligned}
\mathcal{M} \bar{w}+g(\bar{w}) & =\epsilon \bar{w}^{\prime \prime}+J \star \bar{w}-\bar{w}-c \bar{w}^{\prime}+g(\bar{w}) \\
& =\epsilon \delta^{2} e^{-\delta x}+J \star \bar{w}-\left(1-e^{-\delta x}\right)-\delta c e^{-\delta x}+\delta\left(\kappa_{0}-\delta\right) e^{-\delta x} \\
& \leq \epsilon \delta^{2} e^{-\delta x}+1-1+e^{-\delta x}-\delta c e^{-\delta x}+\delta\left(\kappa_{0}-\delta\right) e^{-\delta x} \\
& \leq e^{-\delta x}\left[1-\delta\left(c-\kappa_{0}\right)-\delta^{2}(1-\epsilon)\right] \\
& \leq 0
\end{aligned}
$$

for $c$ large enough, say

$$
c \geq \kappa_{2}=\frac{1+\delta \kappa_{0}-\delta^{2}(1-\epsilon)}{\delta} .
$$

Thus by taking $c \geq \sup \left\{\kappa_{0}, \kappa_{1}, \kappa_{2}\right\}$, we achieve

$$
\begin{array}{r}
g(\bar{w}) \geq f(\bar{w}) \quad \text { and } \quad J \star \bar{w}-\bar{w}-c \bar{w}^{\prime}+g(\bar{w}) \leq 0 \\
\text { for } \quad 0 \leq \bar{w} \leq e^{-\lambda N} \text { and } \quad \bar{w} \geq 1-e^{-\delta N} .
\end{array}
$$

For the remaining values of $\bar{w}$, i.e. for $x \in[-N, N], \bar{w}^{\prime}>0$ and we may increase $c$ further if necessary, to achieve

$$
\epsilon \bar{w}^{\prime \prime}+J \star \bar{w}-\bar{w}-c \bar{w}^{\prime}+g(\bar{w}) \leq 0 \text { in } \mathbb{R} .
$$

The result follows for

$$
\bar{\kappa}(\epsilon):=\sup \left\{\kappa_{0}, \kappa_{1}, \kappa_{2}, \kappa_{3}\right\},
$$

where

$$
\kappa_{3}=\sup _{x \in[-N, N]}\left\{\frac{\epsilon\left|\bar{w}^{\prime \prime}\right|+|J \star \bar{w}-\bar{w}|+g(\bar{w})}{\bar{w}^{\prime}}\right\} .
$$

Remark 2.1. $\bar{\kappa}(\epsilon)$ is a nondecreasing function of $\epsilon$.

Remark 2.2. Observe that given any $r \in \mathbb{R}$, then for $c \geq \bar{\kappa}(\epsilon), \bar{w}$ also satisfies:

$$
\left\{\begin{array}{c}
\mathcal{L} \bar{w}+f(\bar{w}) \leq 0 \quad \text { in } \Omega \\
\bar{w}(-r) \geq 0 \\
\bar{w}(+\infty)=1,
\end{array}\right.
$$

where $\mathcal{L}=\mathcal{L}(\epsilon, c, r)$ is defined by (1.7).

Next, we construct super and subsolutions of (1.5). 
Remark 2.3. Let $\epsilon \geq 0, r \in \mathbb{R}, c \in \mathbb{R}, \theta \in(0,1)$. Then the constant functions $\underline{u}=\theta$ and $\bar{u}=1$ are respectively $a$ sub-and a supersolution of problem (1.5), i.e.

$$
\left\{\begin{array}{cll}
\mathcal{L} \underline{u}+f(\underline{u}) \geq-h_{r}(x) & \text { in } \Omega & \left(\text { resp. } \quad \mathcal{L} \bar{u}+f(\bar{u}) \geq-h_{r}(x) \text { in } \Omega\right), \\
\underline{u}(-r) \leq \theta & (\text { resp. } \bar{u}(-r) \leq \theta), \\
\underline{u}(+\infty) \leq 1, & (\text { resp. } \bar{u}(+\infty) \leq 1) .
\end{array}\right.
$$

We now construct a subsolution of (1.5) satisfying stronger conditions on the boundary of $\Omega$.

\section{Lemma 2.2.}

Let $\epsilon>0, r \in \mathbb{R}, \theta \in(0,1)$. There exists $\underline{\kappa}(\epsilon) \in \mathbb{R}$ and an increasing function $\underline{w} \in C^{2}(\mathbb{R})$ such that, given any $c \leq \underline{\kappa}(\epsilon)$,

$$
\left\{\begin{array}{l}
\mathcal{L} \underline{w}+f(\underline{w}) \geq-h_{r}(x) \text { in } \Omega \\
\underline{w}(-r)=\theta \\
\underline{w}(+\infty)=1
\end{array}\right.
$$

\section{Proof:}

Let $f_{b}$ be a smooth bistable function (i.e. $f_{b}(0)=f_{b}(1)=0$ and $\exists \theta \in(0,1)$ such that $f_{b}<0$ in $(0, \theta), \quad f_{b}(\theta)=0$ and $f_{b}>0$ in $\left.(\theta, 1)\right)$ such that $f_{b} \leq f$ and $\int_{0}^{1} f_{b}(s) d s>0$. Let $\left(u_{b}, c_{b}\right)$ denote the unique (up to translation) increasing solution of (1.9) with $f_{b}$ instead of $f$. Such a solution exists, see [1] for details. Moreover $c_{b}>0$. Using the translation invariance of (1.9), one can easily show that for any $c \leq c_{b}, u_{b}^{\tau}:=u_{b}(.+\tau)$ is a subsolution of (1.5) for some $\tau \in \mathbb{R}$. Indeed, choose $\tau$ such that $u_{b}^{\tau}(-r)=\theta$.

Since $u_{b}^{\tau}$ is increasing we have

$$
h_{r}(x)=\theta \int_{-\infty}^{-r} J(x-y) d y \geq \int_{-\infty}^{-r} J(x-y) u_{b}^{\tau}(y) d y .
$$

A simple computation shows that

$$
\begin{aligned}
\mathcal{L} u_{b}^{\tau}+h_{r}(x)+f\left(u_{b}^{\tau}\right) & \geq \mathcal{L} u_{b}^{\tau}+\int_{-\infty}^{-r} J(x-y) u_{b}^{\tau}(y) d y+f_{b}\left(u_{b}^{\tau}\right) & & \text { in } \Omega \\
& \geq \mathcal{M} u_{b}^{\tau}+f_{b}\left(u_{b}^{\tau}\right)=\left(c_{b}-c\right)\left(u_{b}^{\tau}\right)^{\prime} & & \text { in } \Omega .
\end{aligned}
$$

Hence for $c \leq c_{b}$,

$$
\left\{\begin{array}{l}
\mathcal{L} u_{b}^{\tau}+h_{r}(x)+f\left(u_{b}^{\tau}\right) \geq\left(c_{b}-c\right)\left(u_{b}^{\tau}\right)^{\prime} \geq 0 \text { in } \Omega \\
\\
u_{b}^{\tau}(-r)=\theta \\
u_{b}^{\tau}(+\infty)=1
\end{array}\right.
$$

\section{$3 \quad L^{2}$ estimates}

In this Section, we obtain $L^{2}$ estimates for solutions $u$ of the problems (1.5) and (1.9). 


\section{1 $\quad L^{2}$ estimates for solutions of (1.9)}

\section{Lemma 3.1.}

Assume $\epsilon>0, c \in \mathbb{R}$ and let $u$ be a smooth increasing solution of (1.9) then

(i) $u^{\prime}, u^{\prime \prime} \in L^{2}(\mathbb{R})$

(ii) $1-u \in L^{2}\left(\mathbb{R}^{+}\right)$.

\section{Proof of Lemma 3.1:}

Let $u$ be a smooth increasing solution of (1.9). We start out by showing that $u^{\prime}$ and $u^{\prime \prime}$ vanish at infinity. We restrict to the case $u^{\prime}(+\infty)=0$, the other cases $u^{\prime}(-\infty)=0$ and $u^{\prime \prime}( \pm \infty)=0$ being similar.

Assume by contradiction that there exists an increasing sequence $\left(x_{p}\right)_{p \in \mathbb{N}}$ converging to $+\infty$ and $\alpha>0$ such that

$$
\forall p \in \mathbb{N} \quad u^{\prime}\left(x_{p}\right) \geq \alpha .
$$

Let $\left(u_{p}\right)_{p \in \mathbb{N}}$ defined by

$$
u_{p}(x):=u\left(x+x_{p}\right) \quad \text { for } \quad x \in \mathbb{R} .
$$

Clearly, $u_{p}$ solves (1.9) and $0 \leq u_{p} \leq 1$. By definition of $\left(u_{p}\right)_{p \in \mathbb{N}}$, we have on every compact set,

$$
\lim _{p \rightarrow+\infty} u_{p}(x) \equiv 1 \text {. }
$$

Since $u_{p}$ satisfies (1.9), using standard elliptic estimates, $u_{p} \rightarrow 1$ in $C_{l o c}^{2, \beta}$. In particular, $u_{p} \rightarrow 1$ in $C^{2, \beta}(-1,1)$ and $u_{p}^{\prime}(0) \rightarrow 0$ as $p$ goes to $+\infty$. Using now (3.1), we have

$$
0<\alpha \leq u^{\prime}\left(x_{p}\right)=u_{p}^{\prime}(0) \rightarrow 0,
$$

which is our desired contradiction.

We show next that $f(u) \in L^{1}(\mathbb{R})$. Integrating (1.9) over $(-r, r)$ leads to:

$$
\epsilon\left(u^{\prime}(r)-u^{\prime}(-r)\right)+\int_{-r}^{r}(J \star u-u) d x-c(u(r)-u(-r))=-\int_{-r}^{+r} f(u) .
$$

Assume for the moment that $J \star u-u \in L^{1}(\mathbb{R})$, then we can pass to the limit as $r \rightarrow+\infty$ in the above expression. So we get

$$
\int_{-\infty}^{+\infty}(J \star u-u) d x-c=-\int_{-\infty}^{+\infty} f(u) .
$$

Therefore $f(u) \in L^{1}(\mathbb{R})$, provided that $J \star u-u \in L^{1}(\mathbb{R})$.

\section{Claim 3.1.}

$J \star u-u \in L^{1}(\mathbb{R})$. Moreover,

$$
\|J \star u-u\|_{L^{1}} \leq \int_{\mathbb{R}} J(z)|z| d z \quad \text { and } \quad \int_{\mathbb{R}}(J \star u-u)=0 .
$$




\section{Proof:}

Clearly,

$$
\int_{-r}^{r}|(J \star u-u)| \leq \int_{-r}^{r} \int_{\mathbb{R}} J(x-y)|u(y)-u(x)| d y d x .
$$

Using the change of variable in $y, z:=y-x$, (3.2) becomes

$$
\int_{-r}^{r}|(J \star u-u)| \leq \int_{-r}^{r} \int_{\mathbb{R}} J(z)|u(x+z)-u(x)| d z d x .
$$

Since $u \in C^{1}(\mathbb{R}),|u(x+z)-u(x)|=|z| \int_{0}^{1} u^{\prime}(x+s z) d s$.

Plug this equality in (3.3) to obtain:

$$
\int_{-r}^{r} \int_{\mathbb{R}} J(z)|u(x+z)-u(x)| d y d x=\int_{-r}^{r} \int_{\mathbb{R}} J(z)|z| \int_{0}^{1} u^{\prime}(x+s z) d s d z d x .
$$

Since all terms are positive, using Tonnelli's Theorem, we can permute the order of integration and obtain

$$
\begin{aligned}
\int_{-r}^{r} \int_{\mathbb{R}} J(z)|z| \int_{0}^{1} u^{\prime}(x+s z) d s d z d x & =\int_{\mathbb{R}} J(z)|z| \int_{-r}^{r} \int_{0}^{1} u^{\prime}(x+s z) d s d x d z \\
& =\int_{0}^{1} \int_{\mathbb{R}} J(z)|z|[u(r+s z)-u(-r+s z)] d z d s .
\end{aligned}
$$

Hence,

$$
\int_{-r}^{r}\left|\int_{\mathbb{R}} J(x-y)(u(y)-u(x)) d y\right| d x \leq \int_{0}^{1} \int_{\mathbb{R}} J(z)|z|[u(r+s z)-u(-r+s z)] d z d s .
$$

Using now Lebesgue dominated convergence, we can pass to the limit in the above expression to get

$$
\|J \star u-u\|_{L^{1}} \leq \int_{\mathbb{R}} J(z)|z| d z .
$$

Let us now compute $\int_{\mathbb{R}}(J \star u-u) d x$. Since $J$ is symmetric, we have

$$
\begin{aligned}
\int_{\mathbb{R}}(J \star u-u) d x & =\int_{\mathbb{R}^{2}} J(x-y)(u(y)-u(x)) d y d x \\
& =\int_{\mathbb{R}^{2}} J(y-x)(u(y)-u(x)) d y d x=\int_{\mathbb{R}^{2}} J(x-y)(u(x)-u(y)) d y d x .
\end{aligned}
$$

Hence,

$$
2 \int_{\mathbb{R}^{2}} J(x-y)(u(y)-u(x)) d y d x=0
$$


We now prove (i). Multiplying (1.9) by $u$ and integrating over $\mathbb{R}$ yields

$$
\epsilon \int_{\mathbb{R}} u^{\prime \prime} u+\int_{\mathbb{R}}(J \star u-u) u-c \int_{\mathbb{R}} u^{\prime} u=-\int_{\mathbb{R}} f(u) u .
$$

Integrating by parts the first term yields

$$
-\epsilon \int_{\mathbb{R}}\left(u^{\prime}\right)^{2}+\int_{\mathbb{R}}(J \star u-u) u-\frac{c}{2}=-\int_{\mathbb{R}} f(u) u .
$$

Since $u$ is bounded and $f(u), J \star u-u \in L^{1}$, we conclude that $u^{\prime} \in L^{2}$.

We obtain $u^{\prime \prime} \in L^{2}$ similarly. Indeed, multiplying (1.9) by $u^{\prime \prime}$ and integrating over $\mathbb{R}$ we get

$$
\epsilon \int_{\mathbb{R}}\left(u^{\prime \prime}\right)^{2}+\int_{\mathbb{R}}(J \star u-u) u^{\prime \prime}-c \int_{\mathbb{R}} u^{\prime} u^{\prime \prime}=\int_{\mathbb{R}} f(u) u^{\prime \prime} .
$$

Integration by parts and uniform bounds yield

$$
\begin{aligned}
\epsilon \int_{\mathbb{R}}\left(u^{\prime \prime}\right)^{2} & =-\int_{\mathbb{R}}(J \star u-u) u^{\prime \prime}-\int_{\mathbb{R}} f(u) u^{\prime \prime} \\
& =\int_{\mathbb{R}}\left(J \star u^{\prime}-u^{\prime}\right) u^{\prime}+\int_{\mathbb{R}} f^{\prime}(u)\left(u^{\prime}\right)^{2} \\
& \leq C_{0} \int_{\mathbb{R}} u^{\prime}+C_{1}\left\|u^{\prime}\right\|_{L^{2}(\mathbb{R})}^{2},
\end{aligned}
$$

where $C_{0}$ and $C_{1}$ are positive constants. This ends the proof of (i).

We can now show that $1-u \in L^{2}\left(\mathbb{R}^{+}\right)$. Again multiplying (1.9) by $1-u$ and integrating over $\mathbb{R}$ yields to

$$
\epsilon \int_{\mathbb{R}}\left(u^{\prime}\right)^{2}-\int_{\mathbb{R}}(J \star u-u) u-c / 2+\int_{\mathbb{R}} f(u)(1-u)=0 .
$$

Using now Claim 3.1 and choosing $R$ so large that $f(u) \geq \frac{\left|f^{\prime}(1)\right|}{2}(1-u)$ on $[R, \infty)$, we achieve

$$
\frac{\left|f^{\prime}(1)\right|}{2} \int_{R}^{\infty}(1-u)^{2} \leq \int_{-\infty}^{\infty} f(u)(1-u) \leq C\left(\left\|u^{\prime}\right\|_{L^{2}(\mathbb{R})}^{2}+1\right)<\infty
$$

which proves (ii).

Remark 3.1. Note that these estimates easily extend to solutions of a bistable problem.

Finally, we obtain some useful $L^{2}$ estimates on $J \star u-u$. Namely, we have

\section{Lemma 3.2.}

$$
\|J \star u-u\|_{L^{2}} \leq C\left\|u^{\prime}\right\|_{L^{2}}
$$




\section{Proof:}

Using the Fundamental Theorem of Calculus, we have

$$
\begin{aligned}
\int_{-\infty}^{+\infty} J(x-y) u(y) d y-u(x) & =\int_{-\infty}^{+\infty} J(x-y)(u(y)-u(x)) d y \\
& =\int_{-\infty}^{+\infty} J(z) z\left(\int_{0}^{1} u^{\prime}(x+t z) d t\right) d z .
\end{aligned}
$$

By the Cauchy-Schwartz inequality, it follows that

$$
\begin{aligned}
\left|\int_{-\infty}^{+\infty} J(x-y) u(y) d y-u(x)\right|^{2} & =\left(\int_{-\infty}^{+\infty} J(z) z\left(\int_{0}^{1} u^{\prime}(x+t z) d t\right) d z\right)^{2} \\
& \leq C\left[\int_{-\infty}^{+\infty} \int_{0}^{1} J(z)|z|\left(u^{\prime}\right)^{2}(x+t z) d t d z \cdot \int_{-\infty}^{+\infty} J(z)|z| d z\right] \\
& \leq C^{\prime}\left[\int_{-\infty}^{+\infty} \int_{0}^{1} J(z)|z|\left(u^{\prime}\right)^{2}(x+t z) d t d z\right] .
\end{aligned}
$$

Hence, using Tonnelli's Theorem and a standard change of variables

$$
\begin{aligned}
\int_{-\infty}^{+\infty}\left|\int_{-\infty}^{+\infty} J(x-y) u(y) d y-u(x)\right|^{2} d x & \leq C^{\prime}\left[\int_{-\infty}^{+\infty} \int_{-\infty}^{+\infty} \int_{0}^{1} J(z)|z|\left(u^{\prime}\right)^{2}(x+t z) d t d z d x\right] \\
& \leq C^{\prime \prime} \int_{-\infty}^{+\infty}\left(u^{\prime}\right)^{2}(s) d s
\end{aligned}
$$

Remark 3.2. Lemma 3.1 and Lemma 3.2 imply that $f(u) \in L^{2}(\mathbb{R})$.

\section{2 $L^{2}$ estimates for solutions of (1.5)}

\section{Lemma 3.3.}

Let $\epsilon>0, r \in \mathbb{R}, c \in \mathbb{R}$ and $\theta \in(0,1)$. Let $u$ be a smooth nondecreasing solution of (1.5).

Then

$$
\begin{aligned}
& \text { (iii) } u^{\prime}, u^{\prime \prime} \in L^{2}(\Omega), \\
& \text { (iv) } 1-u \in L^{2}\left(\mathbb{R}^{+} \cap \Omega\right) \text {. }
\end{aligned}
$$

\section{Proof:}

Following the lines of the proof of Lemma 3.1, one can easily show that $u^{\prime}(+\infty)=u^{\prime \prime}(+\infty)=0$.

Next we show that $f(u) \in L^{1}(\Omega)$. Integrating $(1.5)$ over $(-r, R)$ leads to:

$$
\begin{aligned}
\epsilon\left(u^{\prime}(R)-u^{\prime}(-r)\right)+\int_{-r}^{R}\left(\int_{-r}^{+\infty} J(x-y) u(y) d y-u(x)\right) d x & \\
-c(u(R)-u(-r)) & =-\int_{-r}^{R}\left(f(u)-h_{r}(x)\right) d x .
\end{aligned}
$$


Assume for the moment that $\int_{-r}^{+\infty} J(x-y) u(y) d y-u$ and $h_{r}(x)$ are in $L^{1}(\Omega)$. Then passing to the limit as $R \rightarrow+\infty$, we deduce that $f(u) \in L^{1}(\Omega)$. It remains to prove the following

\section{Claim 3.2.}

$\int_{-r}^{+\infty} J(x-y) u(y) d y-u$ and $h_{r}(x)$ are in $L^{1}(\Omega)$.

\section{Proof:}

Start with $h_{r}(x)$. By definition of $h_{r}(x)$, one has

$$
h_{r}(x)=\theta \int_{-\infty}^{-r-x} J(z) d z=: \theta j(x) .
$$

Since $J \geq 0$ and satisfies (H2), a simple computation shows that for some $\lambda>0$,

$$
|j(x)|=\int_{-\infty}^{-r-x} J(z) d z \leq e^{-\lambda(r+x)} \int_{\mathbb{R}} J(z) e^{-\lambda z} d z \leq K e^{-\lambda(r+x)} \in L^{1}(\Omega) .
$$

Now, let us prove that $\int_{-r}^{+\infty} J(x-y) u(y) d y-u \in L^{1}(\Omega)$.

Since $u$ is smooth, using uniform bounds and the Fundamental Theorem of Calculus, we have

$$
\begin{aligned}
\left|\int_{-r}^{+\infty} J(x-y) u(y) d y-u(x)\right| & =\left|\int_{-r}^{+\infty} J(x-y)(u(y)-u(x)) d y-u(x) \int_{-\infty}^{-r} J(x-y) d y\right| \\
& \leq\left|\int_{-r-x}^{+\infty} J(z)(u(x+z)-u(x)) d z\right|+u(x) \int_{-\infty}^{-r-x} J(z) d z \\
& \leq \int_{-r-x}^{+\infty} J(z)|z|\left(\int_{0}^{1} u^{\prime}(x+t z) d t\right) d z+j(x) .
\end{aligned}
$$

Since $j \in L^{1}(\Omega)$, we only need to prove that

$$
\Gamma(x):=\int_{-r-x}^{+\infty} J(z)|z|\left(\int_{0}^{1} u^{\prime}(x+t z) d t\right) d z \in L^{1}(\Omega) .
$$

Integrating $\Gamma$ over $(-r, R)$ yields

$$
\begin{aligned}
\int_{-r}^{R} \Gamma(x) d x & =\int_{-r}^{R} \int_{-r-x}^{+\infty} J(z)|z| \int_{0}^{1} u^{\prime}(x+t z) d t d z d x \\
& =\int_{-r}^{R} \int_{0}^{+\infty} J(z)|z| \int_{0}^{1} u^{\prime}(x+t z) d t d z d x+\int_{-r}^{R} \int_{-r-x}^{0} J(z)|z| \int_{0}^{1} u^{\prime}(x+t z) d t d z d x .
\end{aligned}
$$

Using Tonnelli's Theorem, we end up with

$$
\begin{aligned}
\int_{-r}^{R} \Gamma(x) d x=\int_{0}^{1} \int_{0}^{+\infty} J(z)|z| & \left(\int_{-r}^{R} u^{\prime}(x+t z) d x\right) d z d t \\
& +\int_{0}^{1} \int_{-r-R}^{0} J(z)|z|\left(\int_{-r-z}^{R} u^{\prime}(x+t z) d x\right) d z d t .
\end{aligned}
$$


Hence, we achieve

$$
\begin{aligned}
\int_{-r}^{R} \Gamma(x) d x=\int_{0}^{1} \int_{0}^{+\infty} & J(z)|z|[u(R+t z)-u(-r+t z)] d z d t \\
& +\int_{0}^{1} \int_{-r-R}^{0} J(z)|z|[u(R+t z)-u(-r+(t-1) z)] d z d t .
\end{aligned}
$$

Since $0 \leq u \leq 1$, we end up with

$$
\int_{-r}^{R} \Gamma(x) d x \leq 2 \int_{-\infty}^{+\infty} J(z)|z| d z
$$

which shows that $\Gamma \in L^{1}(\Omega)$.

To obtain (iii) and (iv), we can then follow the proof of Lemma 3.1.

Finally, we obtain some useful $L^{2}$ estimates on $\int_{-r}^{+\infty} J(x-y) u(y) d y-u$. More precisely we have,

\section{Lemma 3.4.}

$\int_{-r}^{+\infty} J(x-y) u(y) d y-u \in L^{2}(\Omega)$. Moreover,

$$
\left\|\int_{-r}^{+\infty} J(x-y) u(y) d y-u\right\|_{L^{2}(\Omega)} \leq C\left(\left\|u^{\prime}\right\|_{L^{2}(\Omega)}+\|j\|_{L^{2}(\Omega)}\right),
$$

where $j(x):=\int_{-\infty}^{-r-x} J(z) d z$.

\section{Proof:}

Again, using the Fundamental Theorem of Calculus, we have

$$
\int_{-r}^{+\infty} J(x-y) u(y) d y-u(x)=\int_{-r-x}^{+\infty} J(z) z\left(\int_{0}^{1} u^{\prime}(x+t z) d t\right) d z-u(x) j(x) .
$$

By the Young and the Cauchy-Schwartz inequalities, it follows that

$$
\begin{aligned}
\left|\int_{-r}^{+\infty} J(x-y) u(y) d y-u(x)\right|^{2} & \leq 2\left[\left(\int_{-r-x}^{+\infty} J(z) z\left(\int_{0}^{1} u^{\prime}(x+t z) d t\right) d z\right)^{2}+u^{2} j^{2}\right] \\
& \leq 2\left[\int_{-r-x}^{+\infty} \int_{0}^{1} J(z)|z|\left(u^{\prime}\right)^{2}(x+t z) d t d z \cdot \int_{-r-x}^{+\infty} J(z)|z| d z+u^{2} j^{2}\right] \\
& \leq C\left[\int_{-r-x}^{+\infty} \int_{0}^{1} J(z)|z|\left(u^{\prime}\right)^{2}(x+t z) d t d z+u^{2} j^{2}\right]
\end{aligned}
$$

Define $\Gamma_{1}(x):=\int_{-r-x}^{+\infty} \int_{0}^{1} J(z)|z|\left(u^{\prime}\right)^{2}(x+t z) d t d z$. We then have 


$$
\left|\int_{-r}^{+\infty} J(x-y) u(y) d y-u(x)\right|^{2} \leq C\left[\Gamma_{1}(x)+j^{2}(x)\right] .
$$

By (3.10), $j \in L^{2}(\Omega)$. Therefore to complete the proof, it remains to show that $\Gamma_{1}$ is in $L^{1}(\Omega)$ and satisfies

$$
\left\|\Gamma_{1}\right\|_{L^{1}(\Omega)} \leq C\left\|u^{\prime}\right\|_{L^{2}(\Omega)}^{2} .
$$

Using Tonelli's Theorem,

$$
\begin{aligned}
\int_{-r}^{R} \Gamma_{1}(x) d x=\int_{0}^{+\infty} J(z)|z| & \left(\int_{-r}^{R} \int_{0}^{1}\left(u^{\prime}\right)^{2}(x+t z) d t d x\right) d z \\
& +\int_{-r-R}^{0} J(z)|z|\left(\int_{-r-z}^{R} \int_{0}^{1}\left(u^{\prime}\right)^{2}(x+t z) d t d x\right) d z .
\end{aligned}
$$

Using a standard change of variables we get

$$
\begin{aligned}
\int_{-r}^{R} \Gamma_{1}(x) d x=\int_{0}^{+\infty} J(z)|z| & \left(\int_{0}^{1} \int_{-r+t z}^{R+t z}\left(u^{\prime}\right)^{2}(s) d s d t\right) d z \\
& +\int_{-r-R}^{0} J(z)|z|\left(\int_{0}^{1} \int_{-r+(t-1) z}^{R+t z}\left(u^{\prime}\right)^{2}(s) d s d t\right) d z .
\end{aligned}
$$

Since $u^{\prime} \in L^{2}(\Omega)$ we then have

$$
\begin{aligned}
\int_{-r}^{R} \Gamma_{1}(x) d x \leq \int_{0}^{+\infty} J(z)|z|\left(\int_{0}^{1} \int_{-r}^{+\infty}\left(u^{\prime}\right)^{2}(s) d s d t\right) d z \\
\quad+\int_{-r-R}^{0} J(z)|z|\left(\int_{0}^{1} \int_{-r}^{+\infty}\left(u^{\prime}\right)^{2}(s) d s d t\right) d z .
\end{aligned}
$$

Hence,

$$
\int_{-r}^{+\infty} \Gamma_{1}(x) d x \leq\left(\int_{-\infty}^{+\infty} J(z)|z| d z\right)\left\|u^{\prime}\right\|_{L^{2}(\Omega)}^{2}
$$

which is the desired conclusion.

\section{Construction of a solution of (1.5)}

In this section, we show that for any fixed $r>0, c \in \mathbb{R}, \epsilon>0$ and for any $\theta \in(0,1)$ there exists a unique increasing solution $u_{r}$ of Problem (1.5). More precisely we show

\section{Theorem 4.1.}

Let $\epsilon>0, r>0, c \in \mathbb{R}$ and $\theta \in(0,1)$. Then there exists a unique smooth increasing solution of (1.5).

We only prove the existence. For the proof of uniqueness, see [6]. 


\subsection{Preliminaries}

Let $G$ be a smooth nondecreasing function such that $G(-r)=\theta, \mathcal{L} G \in L^{2}(\Omega)$ and $1-G \in L^{2}(\Omega)$. For $\lambda>0$, define

$$
\begin{array}{ccc}
T_{\lambda, r}: \quad C_{0}(\Omega) \cap L^{2}(\Omega) & \rightarrow & C_{0}(\Omega) \cap L^{2}(\Omega) \\
v & \mapsto & z,
\end{array}
$$

where $z$ is the unique solution of

$$
\left\{\begin{array}{l}
\mathcal{L} z-\lambda z=F(v, x) \quad \text { in } \Omega, \\
z(-r)=0 \\
z(+\infty)=0
\end{array}\right.
$$

where $F(v, x)=-f(v+G)-\lambda v-\mathcal{L} G-h_{r}(x)$. Using Lemma A.1, to prove that $z$ is well-defined, it is enough to show that

$$
v \in L^{2}(\Omega) \cap C_{0}(\Omega) \Longrightarrow F(v, x) \in L^{2}(\Omega) \cap C_{0}(\Omega) .
$$

By definition of $G, \mathcal{L} G \in L^{2}(\Omega)$. By (3.10), $h_{r} \in L^{2}(\Omega)$. So we are left to prove that $f(v+G) \in L^{2}(\Omega)$.

Given $v \in L^{2}(\Omega) \cap C_{0}(\Omega)$, since $f(1)=0$ and $1-G \in L^{2}(\Omega)$,

$$
|f(v+G)| \leq\left\|f^{\prime}\right\|_{\infty}|v+G-1| \in L^{2}(\Omega) \quad \text { and } \quad \lim _{+\infty} f(v+G)=0 .
$$

Hence, $f(v+G) \in L^{2}(\Omega) \cap C_{0}(\Omega)$.

\subsection{Iteration procedure}

We claim that there exists a sequence of functions $\left(u_{n}\right)_{n \in \mathbb{N}}$ satisfying

$$
\begin{gathered}
u_{0}=G \text { and for } n \in \mathbb{N} \backslash\{0\}, \\
\left\{\begin{array}{l}
\mathcal{L} u_{n+1}-\lambda u_{n+1}=-f\left(u_{n}\right)-\lambda u_{n}-h_{r}(x) \quad \text { in } \Omega, \\
u_{n+1}(-r)=\theta, \\
u_{n+1}(+\infty)=1 .
\end{array}\right.
\end{gathered}
$$

We proceed as follows. Using the substitution $v_{n}=u_{n}-G,(4.2)$ reduces to

$$
\left\{\begin{array}{l}
\mathcal{L} v_{n+1}-\lambda v_{n+1}=F\left(v_{n}, x\right) \quad \text { in } \Omega, \\
v_{n+1}(-r)=0 \\
v_{n+1}(+\infty)=0
\end{array}\right.
$$

where $F(v, x)=-f(v+G)-\lambda v-\mathcal{L} G-h_{r}(x)$. Therefore we want $v_{n+1}=T_{\lambda, r} v_{n}$. Using Subsection 4.1 and induction, the sequence $\left(v_{n}\right)_{n \in \mathbb{N}}$ is well defined provided that $v_{0} \in L^{2}(\Omega) \cap C_{0}(\Omega)$. This is trivial since $v_{0}=0$.

Remark 4.1. Observe that if $u_{0}$ is a supersolution (resp. a subsolution) of (1.5) and if $\lambda$ is chosen so large that $-f-\lambda$ is nonincreasing, the Maximum Principle (Theorem A.2) implies that $\left(u_{n}\right)_{n \in \mathbb{N}}$ is nonincreasing (resp. nondecreasing). 


\subsection{Passing to the limit as $n \rightarrow \infty$}

Assume that $u_{0}$ is either a supersolution or a subsolution satisfying $\theta \leq u_{0} \leq 1$. Recall that the constants $\theta$ and 1 are respectively a subsolution and a supersolution of (1.5).

It follows easily by induction and the Maximum Principle (Theorem A.2) that for all $n \in \mathbb{N}$,

$$
\theta \leq u_{n} \leq 1
$$

Choosing $\lambda>0$ so large that $-f-\lambda$ is nonincreasing, we prove next by induction that, given $n \in \mathbb{N}$,

$$
x \rightarrow u_{n}(x) \text { is a nondecreasing function. }
$$

First define

$$
\widetilde{u}_{n}(x):= \begin{cases}\theta & \text { if } x \in \mathbb{R} \backslash \Omega, \\ u_{n}(x) & \text { if } x \in \Omega .\end{cases}
$$

We prove that $\widetilde{u}_{n}$ is nondecreasing, which implies (4.5). Observe that $\widetilde{u}_{n+1}$ solves

$$
\left\{\begin{array}{l}
\mathcal{M} \widetilde{u}_{n+1}-\lambda \widetilde{u}_{n+1}=-(f+\lambda)\left(\widetilde{u}_{n}(x)\right) \text { in } \Omega, \\
\widetilde{u}_{n+1}(-r)=\theta \\
\widetilde{u}_{n+1}(+\infty)=1
\end{array}\right.
$$

For $n=0$, we already know that $\widetilde{u}_{0}$ is nondecreasing. Fix now $n \geq 1$ and assume that $\widetilde{u}_{n-1}$ is nondecreasing. Also, given any positive $\tau$, let $w(x)=\widetilde{u}_{n}(x+\tau)-\widetilde{u}_{n}(x)$. It follows from (4.6) and the assumption that $\widetilde{u}_{n-1}$ and $f+\lambda$ are nondecreasing that

$$
\begin{aligned}
& \mathcal{M} w-\lambda w \leq 0 \quad \text { in } \Omega, \\
& w(x) \geq 0 \text { for } \quad x \in \mathbb{R} \backslash \Omega, \\
& w(+\infty)=0
\end{aligned}
$$

whence by the Maximum Principle, $w \geq 0$. In particular, $\widetilde{u}_{n}(x+\tau)-\widetilde{u}_{n}(x) \geq 0$ for any positive $\tau$. This shows that $\widetilde{u}_{n}$ is nondecreasing.

Using Remark 4.1 and the assumption on $u_{0}$, the sequence $\left(u_{n}\right)_{n \in \mathbb{N}}$ is monotone. Hence, using (4.4), (4.5) and Helly's lemma, it follows that $\left(u_{n}\right)_{n \in \mathbb{N}}$ converges pointwise to a nondecreasing function $u$ satisfying

$$
\theta \leq u \leq 1
$$

By the dominated convergence theorem, we have for all $x \in \Omega$

$$
\int_{-r}^{+\infty} J(x-y) u_{n}(y) d y-u_{n}(x) \rightarrow \int_{-r}^{+\infty} J(x-y) u(y) d y-u(x), \quad \text { as } \quad n \rightarrow \infty .
$$

Rewriting (4.2) as

$$
\epsilon u_{n+1}^{\prime \prime}-c u_{n+1}^{\prime}=u_{n+1}-\int_{-r}^{+\infty} J(x-y) u_{n+1}(y) d y-\lambda\left(u_{n}-u_{n+1}\right)-f\left(u_{n}\right)-h_{r}(x)
$$


observing that the right-hand side in the above equation is uniformly bounded and using elliptic regularity, we conclude that $\left(u_{n}\right)_{n \in \mathbb{N}}$ is bounded e.g. in $C^{1, \alpha}(\omega)$, where $\alpha \in(0,1)$ and $\omega$ is an arbitrary bounded open subset of $\Omega$. Bootstrapping the argument implies that $\left(u_{n}\right)_{n \in \mathbb{N}}$ is bounded in $C^{2, \alpha}(\omega)$. Hence $u \in C^{2}(\Omega)$ and we can pass to the limit in the equation to obtain that $u$ solves

$$
\mathcal{L} u+f(u)+h_{r}(x)=0 \quad \text { in } \Omega
$$

Observing that $u_{n}(-r)=\theta$ and that $\left(u_{n}\right)_{n \in \mathbb{N}}$ converges pointwise to $u$, we easily conclude that $u(-r)=\theta$.

To complete the construction of the solution, we prove that $u(+\infty)=1$. Indeed, since $u$ is uniformly bounded and nondecreasing, $u$ achieves its limit at $+\infty$. Using Lemma 3.3, $u^{\prime}(+\infty)=u^{\prime \prime}(+\infty)=0$. It follows from (4.11) that $f(u(+\infty))=0$. Hence, $u(+\infty)=1$. We have thus constructed an increasing solution $u$ of (1.5), provided we have an adequate sub or supersolution $u_{0}$ of (1.5).

Remark 4.2. In the case where $u_{0}$ is a subsolution of (1.5), one has

$$
u_{0} \leq u \leq 1
$$

Hence, $u(+\infty)=1$ is a direct consequence of $u_{0}(+\infty)=1$.

The construction of a nondecreasing solution of (1.5) is now reduced to finding a good sub or supersolution $u_{0}$ satisfying $u_{0}(-r)=\theta, \mathcal{L} u_{0} \in L^{2}(\Omega)$ and $1-u_{0} \in L^{2}(\Omega)$ for fixed $r>0, \theta \in(0,1), \epsilon>0$ and $c \in \mathbb{R}$.

\subsection{Construction of a solution of (1.5) for $c \leq \underline{\kappa}(\epsilon)$}

Assume that $r>0, \theta \in(0,1), \epsilon>0$ are fixed and let $c \leq \underline{\kappa}(\epsilon)$, where $\underline{\kappa}(\epsilon)$ is given by Lemma 2.2. Recall that $\underline{w}$ given by Lemma 2.2 is a subsolution of (1.5), with $c \leq \underline{\kappa}(\epsilon)$.

Using Lemmas 3.1-3.2 and Remark 3.1 yields

$$
\underline{w}^{\prime \prime}, \underline{w}^{\prime},(J \star \underline{w}-\underline{w}) \in L^{2}(\mathbb{R}) \text { and } 1-\underline{w} \in L^{2}\left(\mathbb{R}^{+}\right)
$$

Hence,

$$
|\mathcal{L} \underline{w}| \leq \epsilon\left|\underline{w}^{\prime \prime}\right|+\left|c \underline{w}^{\prime}\right|+\left|\int_{-\infty}^{+\infty} J(x-y) \underline{w}(y) d y-\underline{w}\right| \in L^{2}(\Omega) .
$$

We then apply the previous subsection with $u_{0}=\underline{w}$ to obtain a nondecreasing solution of (1.5) for $c \leq \underline{\kappa}(\epsilon)$.

\subsection{Construction of a solution for $c>\underline{\kappa}(\epsilon)$}

To obtain solutions for $c>\underline{\kappa}(\epsilon)$, we argue as follows. Assume as in the previous subsection that $r>0, \theta \in(0,1), \epsilon>0$ are fixed and choose $c>\underline{\kappa}(\epsilon)$.

Let $u_{s}$ be the smooth nondecreasing solution of (1.5) obtained with $c=\underline{\kappa}(\epsilon)$. Since $c>\underline{\kappa}(\epsilon)$ and $u_{s}$ is increasing, $u_{s}$ is a supersolution of (1.5) with speed $c$. By 
construction, we have $u_{s} \geq \theta$ and $\theta$ is a subsolution of (1.5). Therefore to obtain a solution of (1.5), it is sufficient to prove that $\mathcal{L} u_{s} \in L^{2}(\Omega)$ and $1-u_{s} \in L^{2}(\Omega)$. The latter is easily obtained using the $L^{2}$ estimates (Lemmas 3.3-3.4) obtained in the previous section.

\section{Construction of solutions of (1.9) for all $c \geq c^{*}(\epsilon)$}

In this section, we study problem (1.9) and prove the following

\section{Theorem 5.1.}

Let $\epsilon>0$, then there exists a positive real number $c^{*}(\epsilon)$ such that for all $c \geq c^{*}(\epsilon)$ there exists a positive smooth increasing solution $u_{\epsilon}$ of (1.9). Furthermore if $c<c^{*}(\epsilon)$, then problem (1.9) has no increasing solution.

The proof of Theorem 5.1 will be split in two parts. In the first part, Subsection 5.1, we construct a solution of Problem (1.9) for a specific value of the speed $c=\bar{\kappa}(\epsilon)$, using solutions of approximate problems constructed in the previous section and a standard limiting procedure. Then in the second part, Subsection 5.2, we define the minimal speed $c^{*}(\epsilon)$ and construct solutions of (1.9) for speeds $c \geq c^{*}(\epsilon)$.

\subsection{Construction of one solution of (1.9) for $c=\bar{\kappa}(\epsilon)$}

In this section, we consider problem (1.5) with $c=\bar{\kappa}(\epsilon)$, where $\kappa(\epsilon)$ is given by Lemma 2.1.

By Theorem 4.1, for any real number $r$ and any $\theta \in(0,1)$ there exists a unique solution of (1.5). For fixed $r>0$, we claim that the solution of (1.5) satisfies the following normalization.

\section{Claim 5.1.}

Fix $\epsilon>0$ and $r>0$. There exists $\theta_{0} \in(0,1)$ such that the corresponding solution $u_{r}^{\theta_{0}}$ of (1.5) with $\theta=\theta_{0}$ satisfies the normalization $u_{r}^{\theta_{0}}(0)=\frac{1}{2}$.

\section{Proof of Claim 5.1}

Define

$$
\Theta=\left\{\theta \mid u_{r}^{\theta}(0)>\frac{1}{2}\right\} .
$$

Choosing any $\theta \geq \frac{1}{2}$ and observing that $u_{r}^{\theta}$ is increasing we have $\left[\frac{1}{2}, 1\right) \subset \Theta$. The uniqueness of the solution $u_{r}^{\theta}$ and standard a priori estimates imply that $\theta \rightarrow u_{r}^{\theta}(0)$ is continuous over $[0,1]$. By continuity, we can therefore conclude that

- Either there exists a positive $\theta_{0}$ such that $u_{r}^{\theta_{0}}(0)=\frac{1}{2}$

- Or $(0,1) \subset \Theta$.

We show that the latter case cannot occur, which proves the claim. For this, we argue by contradiction. Suppose that $(0,1) \subset \Theta$. Let $\left(\theta_{n}\right)_{n \in \mathbb{N}}$ be a sequence such that $\theta_{n} \rightarrow 0$. Let $\left(u_{n}\right)_{n \in \mathbb{N}}$ be the corresponding sequence of solutions of (1.5) with $\theta=\theta_{n}$. Using Helly's Lemma and standard a priori estimates, we can extract a 
subsequence, still denoted $\left(u_{n}\right)_{n \in \mathbb{N}}$ which converges to a nondecreasing function $u$. Clearly, $u(-r)=0$. Since $u_{n}(0)>\frac{1}{2}$, we have $u(0) \geq \frac{1}{2}$. Hence, $u$ is non trivial and satisfies

$$
\left\{\begin{array}{l}
\mathcal{L} u+f(u)=0 \quad \text { for } x \in(-r,+\infty) \\
u(-r)=0 \\
u(+\infty)=1
\end{array}\right.
$$

The fact that $u(+\infty)=1$ is obtained using Lemma 3.3 and following the arguments of Subsection 4.3 .

Observe that $\bar{w}$ given by Lemma 2.1 is a supersolution of (5.1).

One can show that $\bar{w}>u$, (see [6] for details), which provides a contradiction, since $\frac{1}{2} \leq u(0)<\bar{w}(0)=\frac{1}{2}$.

With the latter normalization, we are ready for the construction of a solution of (1.9). Let $\left(r_{n}\right)_{n \in \mathbb{N}}=(n)_{n \in \mathbb{N}}$ and $\left(u_{n}^{\theta_{n}}\right)_{n \in \mathbb{N}}$ be the sequence of solutions of the corresponding approximate problem (1.5) with $r=r_{n}$ and $\theta=\theta_{n}$, where $\left(\theta_{n}\right)_{n \in \mathbb{N}}$ is such that $u_{n}^{\theta_{n}}(0)=\frac{1}{2}$. Define $\left(h_{n}\right)_{n \in \mathbb{N}}$ by

$$
h_{n}(x)=\theta_{n} \int_{-\infty}^{-r_{n}} J(x-y) d y .
$$

By Theorem 4.1 and Claim 5.1 such sequences are well defined.

Clearly, $h_{n} \rightarrow 0$ pointwise, as $n \rightarrow \infty$. Observe now that $\left(u_{n}^{\theta_{n}}\right)_{n \in \mathbb{N}}$ is a uniformly bounded sequence of increasing functions. Therefore using Helly's lemma, there exists a subsequence which converges pointwise to a nondecreasing function $u$. Since $\epsilon>0$, using local $C^{2, \alpha}$ estimates, up to extraction, the subsequence converges in $C_{l o c}^{2, \alpha}$. Therefore $u \in C^{2, \alpha}$ and satisfies

$$
\mathcal{M} u+f(u)=0 \quad \text { in } \mathbb{R} .
$$

From the normalization and the fact that $f\left(\frac{1}{2}\right) \neq 0, u$ is non trivial. Since $u$ is increasing and bounded, $u$ achieves its limits $l^{ \pm}$at $\pm \infty$. A standard argument, using Lemma 3.1, implies that $f\left(l^{ \pm}\right)=0$. Since $l^{-} \leq \frac{1}{2}$ and $l^{+} \geq \frac{1}{2}$, we must have $u(-\infty)=0$ and $u(+\infty)=1$. Therefore we have constructed a non trivial solution of (1.9) for $c=\bar{\kappa}(\epsilon)$.

Remark 5.1. Observe that the existence of a supersolution $\bar{w}$ is only needed in the normalization process. Therefore, the previous construction holds with any other supersolution $\psi$ of (1.9) such that $\psi(0)=\frac{1}{2}$.

Let us now turn our attention to the second part of the proof.

\subsection{Definition of $c^{*}(\epsilon)$}

Define

$$
c^{*}(\epsilon):=\inf \{c>0: \quad(1.9) \text { admits an increasing solution }\}
$$


By the previous section, $c^{*}(\epsilon)$ is well defined. Obviously, from the definition of $c^{*}(\epsilon)$, there is no increasing solution to (1.9) for speeds $c<c^{*}(\epsilon)$. Our goal in this subsection is to provide a solution of (1.9) for all $c \geq c^{*}(\epsilon)$.

First we observe that (1.9) has a solution for $c=c^{*}(\epsilon)$. Let $\left(c_{n}\right)_{n \in \mathbb{N}}$ be a minimizing sequence for $c^{*}(\epsilon)$. The corresponding solutions $u_{n}$ of (1.9) are increasing (and uniformly bounded by 1) so that we may apply Helly's lemma and elliptic regularity as in the previous section to conclude that $\left(u_{n}\right)_{n \in \mathbb{N}}$ converges to an increasing solution of (1.9) for $c=c^{*}(\epsilon)$, which we denote by $u_{\epsilon}$. Boundary conditions for $u_{\epsilon}$ are obtained as in Subsection 5.1 using the fact that $u_{\epsilon}(0)=u_{n}(0)=\frac{1}{2}$.

Fix now $c>c^{*}(\epsilon)$ and observe that $\bar{w}:=u_{\epsilon}$ is a smooth increasing supersolution of (1.9) with speed c. Since $u_{\epsilon}(0)=\frac{1}{2}$, by Remark 5.1, the construction of Subsection 5.1 applies. Therefore, we get a solution of (1.9) for all $c \geq c^{*}(\epsilon)$ which ends the proof of Theorem 5.1 .

\section{Existence of a solution for $\epsilon=0$}

In the previous section, we were able to prove that for every positive $\epsilon$, Problem (1.9) admits a semi infinite interval of solution, i.e for $c \geq c^{*}(\epsilon)$ there exists a positive increasing solution of (1.9). We will see that the same holds true for (1.4). The idea is to let $\epsilon \rightarrow 0$ in (1.9) and to extract a converging sequence of solutions. The main problem is to control $c^{*}(\epsilon)$ when $\epsilon \rightarrow 0$. We prove the following:

Lemma 6.1.

For every positive $\epsilon_{0}$, there exists $\nu_{0}>0$ such that $c^{*}(\epsilon) \leq \nu_{0}$ for all $\epsilon \in\left[0, \epsilon_{0}\right)$.

\section{Proof:}

According to Remark 2.1, $\bar{\kappa}(\epsilon)$ is an nondecreasing function of $\epsilon$, therefore $\bar{\kappa}(\epsilon) \leq \bar{\kappa}\left(\epsilon_{0}\right)$. The conclusion easily follows from the definition of $c^{*}(\epsilon)$, i.e. $c^{*}(\epsilon) \leq \bar{\kappa}(\epsilon)$.

We now derive existence of a solution of (1.4) for every speed $c$ greater than $\nu_{0}$. More precisely we have the following:

\section{Theorem 6.1.}

There exists $\nu_{0}$ such that for every speed $c$ greater than $\nu_{0}$, there exists a solution $u$ with speed $c$ of (1.4).

\section{Proof:}

According to Lemma 6.1, for $\epsilon$ small, say $\epsilon \leq \epsilon_{0}$, equation (1.9) has a solution $u_{\epsilon}$ for every $c$ greater than $\nu_{0}$ and $\epsilon \leq \epsilon_{0}$. Without loss of generality we assume that for all $\epsilon, u_{\epsilon}(0)=\frac{1}{2}$. From standard a-priori estimates, $u_{\epsilon}$ is a bounded smooth increasing function. Let $\epsilon \rightarrow 0$ along a sequence. As in the previous section, uniform $a$ priori estimates and Helly's theorem applied to $u_{\epsilon}$, provide the existence of a monotone increasing solution $u$ of

$$
[J \star u-u]-c u^{\prime}+f(u)=0 \text { in } \mathbb{R} .
$$


The solution cannot be trivial, according to the normalisation $\frac{1}{2}=u_{\epsilon}(0) \rightarrow u(0)$. Boundary conditions are obtained as in Section 5 .

We define another minimal speed

$$
c^{* *}=\inf \left\{c \mid \forall c^{\prime} \geq c \text { (1.4) has a positive increasing solution of speed } c^{\prime}\right\} .
$$

This minimal speed is well defined according to Theorem 6.1.

Remark 6.1. A quick computation shows that

$$
c^{* *} \leq \liminf _{\epsilon \rightarrow 0} c^{*}(\epsilon) .
$$

Nevertheless, to complete the characterization of the set of solutions of (1.4), we have to prove that there exists no travelling-wave solutions of speed $c$ less than $c^{* *}$. In other words, if we define :

$$
c^{*}=\inf \{c \mid \text { (1.4) has a positive increasing solution of speed } c\},
$$

we have to show that $c^{*}=c^{* *}$. Clearly we have $c^{* *} \geq c^{*}$, the main problem is to prove $c^{* *} \leq c^{*}$. This will be done with the help of the monotony of the speed of truncated problems and its continuous behavior at zero. More precisely, consider equation (6.4) below

$$
\left\{\begin{array}{l}
\epsilon u^{\prime \prime}+[J \star u-u]-c u^{\prime}+\left(f \chi_{\theta}\right)(u)=0 \text { in } \mathbb{R} \\
u(-\infty)=0 \\
u(+\infty)=1
\end{array}\right.
$$

where $\epsilon \geq 0, \theta>0$ and $\chi_{\theta}$ is such that

- $\chi_{\theta} \in C_{0}^{\infty}(\mathbb{R})$,

- $0 \leq \chi_{\theta} \leq 1$,

- $\chi_{\theta}(s) \equiv 0$ for $s \leq \theta$ and $\chi_{\theta}(s) \equiv 1$ for $s \geq 2 \theta$.

We have the following existence and uniqueness theorem

\section{Theorem 6.2.}

Let $\epsilon>0$ and $\theta>0$. There exists a unique speed $c=c_{\theta}(\epsilon)$ and, up to translation, a unique smooth increasing function $u_{\theta}$ such that (6.4) holds. Moreover the speed $c_{\theta}(\epsilon)$ is positive and satisfies

$$
\begin{array}{r}
c_{\theta}(\epsilon)<c^{*}(\epsilon) \\
\lim _{\theta \rightarrow 0} c_{\theta}(\epsilon)=c^{*}(\epsilon) .
\end{array}
$$

Remark 6.2. Theorem 6.2 still holds for $\epsilon=0$, with $c^{*}(0):=c^{*}$ (where $c^{*}$ is given by (6.3)). We then designate the corresponding speed for (6.4) by $c_{\theta}:=c_{\theta}(0)$. 
A proof of Theorem 6.2 and Remark 6.2 can be found in [6, 7], so we do not include it. A natural corollary of this theorem is the continuity of the speed $c_{\theta}(\epsilon)$ with respect to $\epsilon$ and $\theta$. Namely, we have

\section{Corollary 6.1.}

Under the assumptions of Theorem 6.2, the mapping

$$
\begin{aligned}
(0,1) \times[0,1] & \rightarrow \mathbb{R}^{+} \\
(\theta, \epsilon) & \mapsto c_{\theta}(\epsilon)
\end{aligned}
$$

is continuous.

Assume Corollary 6.1 holds true. We then conclude that $c^{*}=c^{* *}$. Indeed, assume by contradiction that $c^{*}<c^{* *}$. Then choose $c$ such that $c^{*}<c<c^{* *}$. By Theorem 6.2 and Remark 6.2, since $c_{\theta}<c^{*}$ for every positive $\theta$, we have $c_{\theta}<c^{*}<c$. Fix $\theta>0$ : since $c_{\theta}(\epsilon)$ is a continuous function of $\epsilon$, one has on the one hand $c_{\theta}(\epsilon)<c$ for $\epsilon$ small, say $\epsilon \in\left[0, \epsilon_{0}\right]$. On the other hand, according to Remark 6.1, we may achieve,

$$
c_{\theta}(\epsilon)<c<c^{*}(\epsilon) \forall \epsilon \in\left[0, \epsilon_{0}\right] .
$$

From this last inequality, and according to (6.6), for each $\epsilon \in\left(0, \epsilon_{0}\right]$ there exists a positive $\theta(\epsilon) \leq \theta$ such that $c=c_{\theta(\epsilon)}(\epsilon)$. Let $u_{\theta(\epsilon)}$ be the associated solution, normalized by $u_{\theta(\epsilon)}(0)=1 / 2$.

Now we take a sequence $\left(\theta_{n}\right)$ converging to 0 . From the above construction, for each $n$ there exists $\epsilon_{n} \leq \theta_{n}$ and $\theta\left(\epsilon_{n}\right) \leq \theta_{n}$ such that $c=c_{\theta\left(\epsilon_{n}\right)}\left(\epsilon_{n}\right)$ and $u_{\theta\left(\epsilon_{n}\right)}$ is the corresponding normalized solution. By construction we have

$$
\theta\left(\epsilon_{n}\right) \rightarrow 0
$$

Use now, as usual, uniform a priori estimates and Helly's theorem to get a solution $\bar{u}$ of (1.4) with speed $c$.

Since $c \in\left(c^{*}, c^{* *}\right)$ is arbitrary, there exists a non trivial solution of (1.4) for any speed $c>c^{*}$, which contradicts the definition of $c^{* *}$. We summarize the above proof in the following diagram :

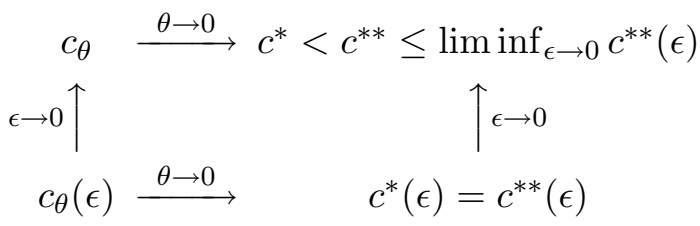

We are left with establishing the

\section{Proof of Corollary 6.1}

We know from Theorem 6.2 and Remark 6.2 that for every $\epsilon \geq 0$ and $\theta>0$ there exists a unique solution $\left(u_{\theta}^{\epsilon}, c_{\theta}(\epsilon)\right)$ of (6.4).

Fix $\epsilon_{0} \geq 0$ and $\theta_{0}>0$. We want to show that for any sequence $\left(\epsilon_{n}, \theta_{n}\right) \rightarrow\left(\epsilon_{0}, \theta_{0}\right)$, we have $c_{\theta_{n}}\left(\epsilon_{n}\right) \rightarrow c_{\theta_{0}}\left(\epsilon_{0}\right)$. 
Let $u_{\theta_{n}}^{\epsilon_{n}}$ be the normalized associated solution, i.e $u_{\theta_{n}}^{\epsilon_{n}}(0)=\frac{1}{2}$. Since $c_{\theta}(\epsilon)>0$ and since (6.5) holds, we have $\left(c_{\theta_{n}}\left(\epsilon_{n}\right)\right)$ bounded as $\left(\epsilon_{n}, \theta_{n}\right) \rightarrow\left(\epsilon_{0}, \theta_{0}\right)$. We can extract a sequence of speeds, which converges to some value $\gamma$. From the a priori estimates on $\left(u_{\theta_{n}}^{\epsilon_{n}}\right)_{n \in \mathbb{N}}$, there also exists a subsequence which converges to a smooth function $u$ solution of the following problem with speed $\gamma$.

$$
\left\{\begin{array}{l}
\epsilon_{0} u^{\prime \prime}+[J \star u-u]-\gamma u^{\prime}+f_{\theta_{0}}(u)=0 \text { in } \mathbb{R} \\
u(-\infty)=0 \\
u(+\infty)=1 .
\end{array}\right.
$$

According to Theorem 6.2, the speed and the profile are unique. Therefore, $\gamma=c_{\theta_{0}}\left(\epsilon_{0}\right)$. Since $\left(c_{\theta_{n}}\left(\epsilon_{n}\right)\right)$ is precompact and has a unique accumulation point, the whole sequence $\left(c_{\theta_{n}}\left(\epsilon_{n}\right)\right)$ must converge to $c_{\theta_{0}}\left(\epsilon_{0}\right)$. This ends the proof of the continuity and by means the characterization of the minimal speed $c^{*}$.

\section{Asymptotic behavior of solutions}

In this section we establish the asymptotic behavior of the solution $u$ near $\pm \infty$ provided $J$ satisfies $(H 2)$. The behavior of the function near $+\infty$ has been already obtained in a previous work by one of the authors [6], therefore we only deal with the behavior of $u$ near $-\infty$.

Remark 7.1. The behavior of $u$ near $\pm \infty$ for bistable and ignition type nonlinearities was also obtained in [6].

We use the same strategy as in [2] and start by proving the following lemma

Lemma 7.1. Assume that (H1) and (H2) hold. Also assume that $f$ is monostable and $f^{\prime}(0)>0$. Let $u$ be an increasing solution of (1.4). Then there exists $\beta>0$ such that

$$
\int_{-\infty}^{\infty} u(x) e^{-\beta x} d x<\infty
$$

\section{Proof}

Let $\zeta \in C^{\infty}(\mathbb{R})$ be a nonnegative nondecreasing function such that $\zeta \equiv 0$ in $(-\infty,-2]$ and $\zeta \equiv 1$ in $[-1, \infty)$. For $N \in \mathbb{N}$, let $\zeta_{N}=\zeta(x / N)$. Multiplying (1.4) by $e^{-\beta x} \zeta_{N}$ and integrating over $\mathbb{R}$, we get

$$
\int(J \star u-u)\left(e^{-\beta x} \zeta_{N}\right)-\int c u^{\prime}\left(e^{-\beta x} \zeta_{N}\right)+\int f(u)\left(e^{-\beta x} \zeta_{N}\right)=0
$$


Since $J$ is even,

$$
\begin{aligned}
\int(J \star u-u)\left(e^{-\beta x} \zeta_{N}\right) & =\int\left(J \star\left(e^{-\beta x} \zeta_{N}\right)-e^{-\beta x} \zeta_{N}\right) u \\
& =\int u(x) e^{-\beta x}\left(\int J(y) e^{\beta y} \zeta_{N}(x-y) d y-\zeta_{N}(x)\right) d x \\
& =\int u(x) e^{-\beta x}\left(\int J(y) e^{-\beta y} \zeta_{N}(x+y) d y-\zeta_{N}(x)\right) d x \\
& \geq \int u(x) e^{-\beta x}\left(\int_{-R}^{\infty} J(y) e^{-\beta y} d y \zeta_{N}(x-R)-\zeta_{N}(x)\right) d x
\end{aligned}
$$

where we used the monotone behaviour of $\zeta_{N}$ in the last inequality and where $R>0$ is chosen as follows : first pick $0<\alpha<f^{\prime}(0)$ and $R>0$ so large that

$$
f(u)(x) \geq \alpha u(x) \quad \text { for } x \leq-R .
$$

Next, one can increase $R$ further if necessary so that $\int_{-R}^{\infty} J(y) d y>(1-\alpha / 2)$. By continuity we obtain for some $\beta_{0}>0$ and all $0<\beta<\beta_{0}$,

$$
\int_{-R}^{\infty} J(y) e^{-\beta y} d y \geq(1-\alpha / 2) e^{\beta R}
$$

Collecting (7.2) and (7.4), we then obtain

$$
\begin{aligned}
\int(J \star u-u)\left(e^{-\beta x} \zeta_{N}\right) & \geq \int u(x) e^{-\beta x}\left((1-\alpha / 2) e^{\beta R} \zeta_{N}(x-R)-\zeta_{N}(x)\right) d x \\
& \geq(1-\alpha / 2) \int u(x+R) e^{-\beta x} \zeta_{N}(x) d x-\int u(x) e^{-\beta x} \zeta_{N}(x) d x \\
& \geq-\alpha / 2 \int u(x) e^{-\beta x} \zeta_{N}(x) d x
\end{aligned}
$$

where we used the monotone behaviour of $u$ in the last inequality.

We now estimate the second term in (7.1):

$$
\begin{aligned}
\int u^{\prime} \zeta_{N} e^{-\beta x} d x & =\beta \int u \zeta_{N} e^{-\beta x}-\int u \zeta_{n}^{\prime} e^{-\beta x} d x \\
& \leq \beta \int u \zeta_{N} e^{-\beta x} .
\end{aligned}
$$

Finally using (7.3), the last term in (7.1) satisfies

$$
\int f(u) \zeta_{N} e^{-\beta x} d x \geq \alpha \int_{-\infty}^{-R} u \zeta_{N} e^{-\beta x} d x-C .
$$

By (7.1), (7.5), (7.6) and (7.7) we then obtain 


$$
(\alpha / 2-c \beta) \int_{-\infty}^{-R} u \zeta_{N} e^{-\beta x} d x \leq C .
$$

Choosing $\beta<\alpha /(2 c)$ and letting $N \rightarrow \infty$ proves the lemma.

Using Lemma 7.1 it is now easy to see that $u(x) \leq C e^{\beta x}$ for all $x \in \mathbb{R}$. Suppose indeed this is not the case and let $x_{n} \in \mathbb{R}$ be such that $u\left(x_{n}\right)>n e^{\beta x_{n}}$.

Since $0 \leq u \leq 1$, we may pick a subsequence $\left(x_{n_{k}}\right)_{k \in \mathbb{N}}$ such that $x_{n_{k+1}}<x_{n_{k}}-1$. But since $u$ is nondecreasing,

$$
\begin{aligned}
\int u(x) e^{-\beta x} d x & \geq \sum_{k \geq 1} \int_{x_{n_{k}}}^{x_{n_{k-1}}} u(x) e^{-\beta x} d x \\
& \geq \sum_{k \geq 1} n_{k} \int_{x_{n_{k}}}^{x_{n_{k}-1}} e^{\beta\left(x_{n_{k}}-x\right)} d x \\
& \geq \sum_{k \geq 1} n_{k} / \beta\left(1-e^{-\beta}\right)=\infty .
\end{aligned}
$$

Acknowledgements. We are very thankful to Henri Berestycki who has supported this work through the "Dynamics of reactive fronts" semester organized at the Institut Henri Poincaré in september 2002. We thank Adam Chmaj for pointing out Schumacher's reference. We also thank Augusto Ponce and Pascal Autissier for useful discussion.

\section{A Appendix}

Here we prove some maximum principles and existence results for solutions of linear problems associated to the operator $\mathcal{L}$ defined by (1.7).

Theorem A.1. Strong Maximum Principle for $\mathcal{L}$ Let $\epsilon \geq 0, r>0, c \in \mathbb{R}$ and $\mathcal{L}$ defined by $(1.7)$ on $\Omega=(-r,+\infty)$. Assume further that Int $(\operatorname{supp} J) \cap \Omega^{-} \neq \emptyset$, where $\Omega^{-}=(-r, 0)$.

Let $u \in C^{2}(\Omega) \cap C^{0}(\bar{\Omega})$ satisfy

$$
\mathcal{L} u \geq 0 \text { in } \Omega \quad(\text { resp. } \mathcal{L} u \leq 0 \text { in } \Omega) .
$$

Then u may not achieve a positive maximum (resp. negative minimum) inside $\Omega$ without being constant.

Similarly we have

Theorem A.2. Strong Maximum Principle for $\mathcal{L}+h_{r}(x)$.

Let $\epsilon \geq 0, r>0, c \in \mathbb{R}, \theta \in(0,1)$ and $\mathcal{L}, h_{r}(x)$ defined by $(1.7)$ on $\Omega=(-r,+\infty)$. Assume further that Int $\operatorname{supp} J) \cap \Omega^{-} \neq \emptyset$, where $\Omega^{-}=(-r, 0)$. 
Let $u \in C^{2}(\Omega) \cap C^{0}(\bar{\Omega})$ satisfy

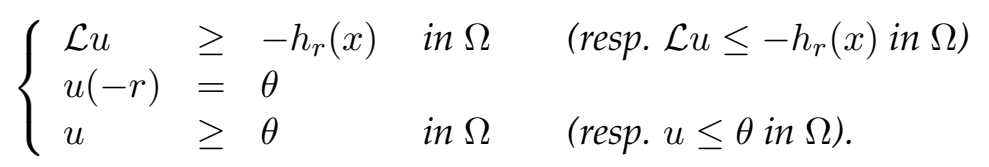

Then u may not achieve a positive maximum (resp. negative minimum) inside $\Omega$ without being constant.

\section{Proof of Theorem A.1:}

We argue by contradiction and assume that $u$ is nonconstant and achieves a positive maximum at some point $x_{0} \in \Omega$. Since $\int_{\mathbb{R}} J(z) d z=1$ we can rewrite (1.7) as

$$
\mathcal{L} u=\epsilon u^{\prime \prime}+\int_{-r}^{+\infty} J(x-y)[u(y)-u(x)] d y-c u^{\prime}-d(x) u,
$$

with $d(x)=\int_{-\infty}^{-r} J(x-y) d y$.

At the point $x_{0}$ of (positive) maximum, we have on the one hand

$$
\epsilon u^{\prime \prime}\left(x_{0}\right) \leq 0, \quad \int_{-r}^{+\infty} J\left(x_{0}-y\right)\left[u(y)-u\left(x_{0}\right)\right] d y \leq 0 \quad \text { and } \quad-d\left(x_{0}\right) u\left(x_{0}\right) \leq 0 .
$$

On the other hand by (A.1),

$$
\epsilon u^{\prime \prime}\left(x_{0}\right)+\int_{-r}^{+\infty} J\left(x_{0}-y\right)\left[u(y)-u\left(x_{0}\right)\right] d y-\bar{d}\left(x_{0}\right) u\left(x_{0}\right) \geq 0
$$

Hence $\epsilon u^{\prime \prime}\left(x_{0}\right)=d\left(x_{0}\right) u\left(x_{0}\right)=0$ and

$$
\int_{-r}^{\infty} J\left(x_{0}-y\right)\left[u(y)-u\left(x_{0}\right)\right] d y=0 .
$$

If $J>0$ in $\mathbb{R}$, we conclude directly that $u(y)=u\left(x_{0}\right)$ for all $y \in \Omega$, contradicting our original assumption.

In general, $J$ is a continuous nonnegative even function with $\operatorname{supp}(J) \cap \Omega^{-} \not \equiv \emptyset$. In particular, there exist constants $0<a<b$ such that $[-b,-a] \cup[a, b] \subset \operatorname{supp}(J)$ and $[a, b] \subset \Omega$. We deduce from (A.6) that

$$
u(y)=u\left(x_{0}\right) \quad \text { for all } y \in\left(x_{0}+[-b,-a] \cup[a, b]\right) \cap \Omega
$$

Let $z=x_{0}+b$ and observe that $u(z)=u\left(x_{0}\right)$. We may thus argue as above and conclude that $u(y)=u(z)$ for all $y \in(z+[-b,-a] \cup[a, b]) \cap \Omega$. In particular,

$$
u(y)=u\left(x_{0}\right) \quad \text { for all } y \in\left(x_{0}+[0, b-a]\right) \cap \Omega .
$$

Repeating the argument with $z=x_{0}+a$, we obtain that $u(y)=u\left(x_{0}\right)$ for all $y \in\left(x_{0}+[-(b-a), 0]\right) \cap \Omega$. Thus,

$$
u(y)=u\left(x_{0}\right) \quad \text { for all } y \in\left(x_{0}+[-(b-a), b-a]\right) \cap \Omega .
$$


Applying the above successively with $x_{0}+b-a$ and $x_{0}-(b-a)$ in place of $x_{0}$, we obtain that $u(y)=u\left(x_{0}\right)$ for all $y \in x_{0}+[-2(b-a), 2(b-a)] \cap \Omega$. Working inductively, we conclude that $u \equiv u\left(x_{0}\right)$ in $\Omega$, which contradicts our original assumption.

\section{Proof of Theorem A.2}

Define

$$
\widetilde{u}(x):= \begin{cases}u(x) & \text { in } \Omega \\ \theta & \text { in } \mathbb{R} \backslash \Omega\end{cases}
$$

and observe that we can rewrite (A.2) as

$$
\begin{cases}\mathcal{M} \widetilde{u} \geq 0 & \text { in } \Omega \\ \widetilde{u}(x) \geq \theta & \text { in } \Omega\end{cases}
$$

where $\mathcal{M} \widetilde{u}=\epsilon \widetilde{u}^{\prime \prime}+[J \star \widetilde{u}-\widetilde{u}]-c \widetilde{u}^{\prime}$.

We argue by contradiction and assume that $\widetilde{u}$ achieves a positive maximum at some point $x_{0} \in \Omega$ and is nonconstant. Since $u(x) \geq \theta$ in $\Omega$ we have $u\left(x_{0}\right)>\theta$. Working as in the proof of Theorem A.1 we obtain that $u \equiv u\left(x_{0}\right)$ on $\bar{\Omega}$, which is a contradiction.

Remark A.1. Theorems $A .2$ and A.1 remain valid when replacing $\mathcal{L}$ by $\mathcal{L}-d_{0}$, where $d_{0}$ is any positive constant.

Next, we provide an elementary lemma to construct solutions of Dirichlet problems associated to $\mathcal{L}$.

Lemma A.1. Let $d_{0}>0, \epsilon>0, r>0, c \in \mathbb{R}$ and $\mathcal{L}$ defined by (1.7) on $\Omega=(-r,+\infty)$. Assume further that Int ( supp $J) \cap \Omega^{-} \neq \emptyset$, where $\Omega^{-}=(-r, 0)$.

Given $f \in C_{0}(\Omega) \cap L^{2}(\Omega)$, there exists a unique solution $u \in C^{2}(\Omega) \cap L^{2}(\Omega)$ of

$$
\left\{\begin{array}{l}
\mathcal{L} u-d_{0} u=f \quad \text { in } \Omega \\
u(-r)=0 \\
u(+\infty)=0
\end{array}\right.
$$

\section{Proof}

Uniqueness follows from the maximum principle. Let $X=H_{0}^{1}(\Omega)$ and define the bilinear form $\mathcal{A}(u, v)$ for $u, v \in X$ by

$\mathcal{A}(u, v)=\epsilon \int_{\Omega} u^{\prime} v^{\prime}+\frac{1}{2} \int_{\Omega} \int_{\Omega} J(x-y)(u(y)-u(x))(v(y)-v(x)) d y d x-c \int_{\Omega} u^{\prime} v+\int_{\Omega} d(x) u v$,

where $d(x)=\int_{-\infty}^{-r} J(x-y) d y+d_{0}$. To solve (A.7), we just need to find $u \in X$ such that $\mathcal{A}(u, v)=\int_{\Omega} u v$ for all $v \in X$. We will show that $\mathcal{A}$ is coercive and continuous in $X$. Existence will then be given by the Lax-Milgram Lemma. Clearly,

$$
\mathcal{A}(u, u) \geq \epsilon \int_{\Omega}\left(u^{\prime}\right)^{2}-c \int_{\Omega} u^{\prime} u+d_{0} \int_{\Omega} u^{2}=\epsilon \int_{\Omega}\left(u^{\prime}\right)^{2}+d_{0} \int_{\Omega} u^{2}
$$


Thus $\mathcal{A}$ is coercive in $X$. It remains to prove the continuity of $\mathcal{A}$. Let $\phi$ and $\psi$ be two smooth functions with compact support in $\Omega$.

$$
|\mathcal{A}(\phi, \psi)| \leq \frac{1}{2} \int_{\mathbb{R}} \int_{\mathbb{R}} J(x-y)|\phi(y)-\phi(x)||\psi(y)-\psi(x)| d y d x
$$

By the Fundamental Theorem of Calculus and the Cauchy-Schwartz inequality we obtain:

$$
\begin{aligned}
|\mathcal{A}(\phi, \psi)| & \leq \int_{\mathbb{R}^{2}} \int_{0}^{1} \int_{0}^{1} J(z) z^{2}\left|\phi^{\prime}(x+t z) \| \psi^{\prime}(x+s z)\right| d z d x d t d s \\
& \leq \int_{\mathbb{R}} \int_{[0,1]^{2}} J(z) z^{2} \int_{\mathbb{R}}\left|\phi^{\prime}(h) \| \psi^{\prime}(h+(s-t) z)\right| d h d s d z d t \\
& \leq \int_{\mathbb{R}} \int_{[0,1]^{2}} J(z) z^{2} d z d t d s\left\|\phi^{\prime}\right\|_{L^{2}(\mathbb{R})}\left\|\psi^{\prime}\right\|_{L^{2}(\mathbb{R})} \\
& \leq\left(\int_{\mathbb{R}} J(z) z^{2} d z\right)\left\|\phi^{\prime}\right\|_{L^{2}(\mathbb{R})}\left\|\psi^{\prime}\right\|_{L^{2}(\mathbb{R})},
\end{aligned}
$$

which shows the continuity of $\mathcal{A}$.

\section{References}

[1] P. W. Bates, P. C. Fife, X. Ren, X. Wang Travelling Waves in a convolution model for phase transition, Arch. Rational Mech. Anal. 138 (1997), 105-136.

[2] H. Berestycki, L. Nirenberg Travelling fronts in cylinder, Ann. Inst. Henri Poincaré 9 (1992), 497-572.

[3] J. Carr, A. Chmaj Uniqueness of travelling waves for nonlocal monostable equations, Preprint

[4] X. Chen Existence, Uniqueness and asymptotic Stability of Travelling Fronts in nonlocal Evolution Equations, Adv. Differential. Equation 2 (1997), 125-160.

[5] J. Coville On the uniqueness and monotonicity of solution of non-local reactiondiffusion equation, to appear in Annali di mathematica pura ed aplicata

[6] J. Coville Équations de réaction-diffusion non-locale, PhD Thesis, Univ. Paris 6, 2003.

[7] J. Coville, L. Dupaigne Min-Max formula for travelling front speed of Non-local reaction-diffusion equation, Nonlinear Analysis: Theory, Methods and Applications Vol. 60-5 pages(797-819).

[8] P. Fife Mathematical aspects of reacting and diffusing systems, Lecture Notes in Biomathematics, 28. Springer-Verlag, Berlin-New York, (1979).

[9] R. A. Fisher, The genetical theory of natural selection, (English. English summary) A complete variorum edition. Revised reprint of the 1930 original. Edited, with a foreword and notes, by J. H. Bennett.Oxford University Press, Oxford, (1999). 
[10] N. Fourier, S. Méléard A microscopic probabilistic description of a locally regulated population and macroscopic approximations Prépublication du Laboratoire de probabilités et modèles aléatoires, 798 (Feb. 2003)

[11] Ya.I. Kanel' Certain problems on equations in the theory of burning, Dokl. Akad. Nauk SSSR 136 277-280 (Russian); translated as Soviet Math. Dokl. 21961 48-51.

[12] A. N. Kolmogorov, I. G. Petrovsky, N. S. Piskunov, Étude de l'équation de la diffusion avec croissance de la quantité de matière et son application à un problème biologique, Bulletin Université d'État à Moscow (Bjul. Moskowskogo Gos. Univ), Série Internationale, Section A. 1, 1937, pp. 1-26, English Translation:Study of the Difussion Equation with Growth of the Quantity of Matter and its Application to a Biological Problem. In Dynamics of curved front, R. PELCÉ Ed., Perspective in Physics Series, Academic Press, New York, (1988), 105-130.

[13] J. D. Murray, Mathematical biology, Third edition. Interdisciplinary Applied Mathematics, 17.Springer-Verlag, New York, (2002).

[14] K. Schumacher Travelling-front solutions for integro-differential equations. I. , J. Reine Angew. Math. 316 (1980), 54-70.

[15] H. F. Weinberger Long-time behavior of a class of biological models, SIAM J. Math. Anal. 13 (1982), no. 3, 353-396.

[16] H. F. Weinberger Asymptotic behavior of a model in population dynamics Nonlinear partial differential equations and applications (Proc. Special Sem., Indiana Univ., Bloomington, Ind., 1976-1977), pp. 47-96. Lecture Notes in Math., Vol. 648, Springer, Berlin, 1978. 